\title{
Bicarbonate Ringer's solution for early resuscitation in hemorrhagic shock rabbits
}

\author{
Long Wang ${ }^{1 \#}$, Jingsheng Lou ${ }^{1 \#}$, Jiangbei Cao ${ }^{1}$, Tao Wang ${ }^{2}$, Jing Liu ${ }^{1}$, Weidong Mi ${ }^{1}$ \\ ${ }^{1}$ Department of Anesthesiology, the First Medical Center of the Chinese People's Liberation Army (PLA) General Hospital, Beijing, China; ${ }^{2}$ New \\ Drug Screening Center/Jiangsu Center for Pharmacodynamics Research and Evaluation, China Pharmaceutical University, Nanjing, China \\ Contributions: (I) Conception and design: J Liu, W Mi; (II) Administrative support: J Liu, W Mi; (III) Provision of study materials or patients: L \\ Wang, T Wang; (IV) Collection and assembly of data: L Wang, T Wang; (V) Data analysis and interpretation: L Wang, J Lou; (VI) Manuscript \\ writing: All authors; (VII) Final approval of manuscript: All authors. \\ \#These authors contributed equally to this work. \\ Correspondence to: Jing Liu; Weidong Mi. Department of Anesthesiology, the First Medical Center of the Chinese People's Liberation Army (PLA) \\ General Hospital, Beijing 100083, China. Email: bj301mzk@163.com; miweidong0709@163.com.
}

Background: Fluid resuscitation is important for correcting hypovolemia. Isotonic crystalloids are the preferred solution for the initial clinical management of patients with multiple traumas. Bicarbonated Ringer's solution (BRS), offering physiological levels of bicarbonate ions and electrolyte ions, can be used for supplementing missing extracellular fluid and correcting metabolic acidosis. We here investigated the effects of BRS on the resuscitation of hemorrhagic shock models and compared the resuscitation performance of three crystalloids, including BRS, acetated Ringer's solution (ARS), and normal saline.

Methods: Thirty adult male New Zealand rabbits were randomly divided into five groups ( $\mathrm{n}=6$ ): a sham operation group (Sham group), an operation without fluid therapy group (Shock group), a BRS group, an ARS group, and a normal saline group (Saline group). The New Zealand rabbits experienced rapid bloodletting to shock status and maintained for 20 minutes except Sham group. The status of shock was maintained in the Shock group. The fluid was infused at a rate of $60 \mathrm{~mL} / \mathrm{kg}$ per hour for 1.5 hours in three fluid therapy group. Measurement of vital signs, arterial blood gas tests, blood biochemistry, hematoxylin and eosin (HE) staining of lung tissue, TUNEL staining of the liver and kidney tissues, and analysis of intestinal flora were performed.

Results: The reduction in both base excess (BE) and bicarbonate ion $\left(\mathrm{HCO}_{3}{ }^{-}\right)$caused by acidosis in rabbits with hemorrhagic shock was significantly improved in the BRS group when compared with the Saline group at infusion for 30 minutes (T3) and 30 minutes after infusion (T5) (BRS group vs. Saline group, BE: at T3, $-4.83 \pm 3.60$ vs. $-12.50 \pm 3.27 \mathrm{mmol} / \mathrm{L}, \mathrm{P}<0.01$; at T5, $-3.67 \pm 4.37$ vs. $-11.00 \pm 2.76 \mathrm{mmol} / \mathrm{L}, \mathrm{P}<0.01 ; \mathrm{HCO}_{3}{ }^{-}$: at T3, $22.15 \pm 2.63$ vs. $15.42 \pm 3.03 \mathrm{mmol} / \mathrm{L}, \mathrm{P}<0.01$; at T5, $23.15 \pm 2.9$ vs. $16.23 \pm 3.07 \mathrm{mmol} / \mathrm{L}, \mathrm{P}<0.01)$. Compared with Shock group, liver cell apoptosis due to hemorrhagic shock was relieved in both the BRS group and ARS group (BRS group vs. Shock group: $19.1 \pm 3.3$ vs. $28.1 \pm 6.1, \mathrm{P}<0.05$; ARS group vs. Shock group: $19.8 \pm 5.4$ vs. $28.1 \pm 6.1, \mathrm{P}<0.05)$.

Conclusions: During resuscitation of hemorrhagic shock, BRS, a novel perioperative balanced crystalloid, is more effective than normal saline in maintaining acid-base balance and in protecting tissues and organs.

Keywords: Traumatic hemorrhagic shock; bicarbonate Ringer's solution (BRS); early fluid resuscitation

Submitted Dec 01, 2020. Accepted for publication Feb 26, 2021.

doi: 10.21037/atm-21-97

View this article at: http://dx.doi.org/10.21037/atm-21-97 


\section{Introduction}

Hemorrhagic shock is one of the main causes of death in trauma patients $(1,2)$. The main pathophysiological change of hemorrhagic shock is a sudden reduction of the effective circulating volume, leading to tissue hypoperfusion, increased anaerobic metabolism, lactic acidosis, reperfusion injury, and endotoxin translocation, which ultimately leads to multiple organ dysfunction syndrome (MODS) (3). Rapid recognition and correction of the cause of circulating volume loss is essential in treating hypovolemic shock. Therefore, fluid resuscitation is also very important for correcting hypovolemia. Isotonic crystalloids are the preferred solution for the initial clinical treatment of patients with multiple traumas (4). The common isotonic crystalloids include normal saline, lactated Ringer's solution (LRS), and acetated Ringer's solution (ARS). Administration of a large amount of normal saline may cause renal injury due to its high chlorine content (5). The lactate in LRS and the acetate in ARS need to be metabolized via the liver $(6,7)$, which may increase the hepatic burden; in addition, the infusion of a large amount of exogenous lactic acid may lead to lactic acid accumulation (8).

Bicarbonate is the main buffer in body. And sodium bicarbonate was the most physiologically alkalinizing reagent and exerted alkalinizing effect by acid-base neutralization, without metabolism. Bicarbonated Ringer's solution (BRS) is the latest generation of balanced crystalloid solutions that contains physiological concentrations of bicarbonate ions and electrolyte ions (sodium ion, potassium ion, calcium ion, magnesium ion and chloride ion), which are more similar to those in the extracellular fluid. Thus, BRS can be used for extracellular fluid resuscitation and for correction of metabolic acidosis. In order to ensure the long-term stability of the coexistence of bicarbonate ions and calcium ions in BRS, citrate was dispensed to chelate calcium ions, and carbon dioxide was filled to maintain the solution in a slightly acidic state, which could inhibit the generation of calcium carbonate. A previous research showed that comparing Ringer's solution (RS) and LRS, BRS was more effective in improving the acidosis in hemorrhagic shock dogs (9). Compared with ARS, BRS was also found to significantly improve the acidbase imbalance in perioperative patients (10). Despite that previous studies about BRS explored its effect on the body internal environment, few studies have explored the effect of resuscitation with different types of crystalloids on the tissues and organs during hemorrhagic shock. We for the first time investigated the resuscitation effects of BRS on internal environment, tissues and organ functions in a surgically established rabbit hemorrhagic shock model, and compared the resuscitation performance of BRS, ARS, and normal saline, with an attempt to inform a better clinical fluid resuscitation options for hemorrhagic shock.

We present the following article in accordance with the ARRIVE reporting checklist (available at http://dx.doi. org/10.21037/atm-21-97).

\section{Methods}

\section{Materials}

The chemical compositions of the three kinds of solution used in this study were shown in Table 1. BRS and ARS were prepared at Hengrui Research Center, Jiangsu Hengrui Pharmaceutical Co., Ltd. (Lianyungang, China). And $0.9 \%$ sodium chloride injection was from Shuanghe Pharmaceutical Co., Ltd. (Anhui, China).

\section{Experimental methods}

\section{Experimental animals and grouping}

Specific pathogen-free male New Zealand rabbits weighing $2.0-2.5 \mathrm{~kg}$ were used for the experiment. The animals were bred in the Experimental Animal Center of China Pharmaceutical University. The animals were accommodated in air-conditioned rooms in which central air-conditioning maintained temperature at a range of $24 \pm$ $2{ }^{\circ} \mathrm{C}$ and a range of relative humidity of $60-80 \%$. The air exchange rate was 10 minutes for external circulation and 5 minutes for internal circulation. The animal rooms were set at 12-hour light-dark cycle. The animals were randomized into Sham group, Shock group, Saline group, ARS group, and BRS group by using the random number table method, with six animals in each group. Number skipping was not allowed during randomization. This study was approved by the Ethical Committee of China Pharmaceutical University, Nanjing University, and Laboratory Animal Management Committee of Jiangsu Province (approval No. CPU-20190723). All of the animal experiments were conducted in compliance with the standard ethical guidelines under the control of the ethical committees mentioned above.

\section{Establishment of animal models}

Adult rabbits were weighed and then intravenously 
Table 1 Compositions of three tested solutions in $\mathrm{mmol} / \mathrm{L}$

\begin{tabular}{|c|c|c|c|c|c|c|c|c|c|}
\hline & $\mathrm{Na}^{+}$ & $\mathrm{K}^{+}$ & $\mathrm{Mg}^{2+}$ & $\mathrm{Ca}^{2+}$ & $\mathrm{Cl}^{-}$ & $\mathrm{HCO}_{3}{ }^{-}$ & Acetate $^{-}$ & Citrate $^{3-}$ & Glucose \\
\hline ARS & 140 & 4 & 1 & 1.5 & 115 & - & 25 & - & 55 \\
\hline BRS & 130 & 4 & 1 & 1.5 & 109 & 28 & - & 1.3 & - \\
\hline
\end{tabular}

BRS, bicarbonated Ringer's solution; ARS, acetated Ringer's solution; $\mathrm{Na}^{+}$, sodium ion; $\mathrm{K}^{+}$, potassium ion; $\mathrm{Mg}^{2+}$, magnesium ion; $\mathrm{Ca}^{2+}$, calcium ion; $\mathrm{Cl}^{-}$, chloride ion; $\mathrm{HCO}_{3}^{-}$, bicarbonate ion.

injected with urethane $(1.0 \mathrm{~g} / \mathrm{kg})$ for general anesthesia. Each animal was fixed on the examination table in a supine position to remove the hair on surgical areas. A median neck incision (about $8 \mathrm{~cm}$ in length) was created under the thyroid cartilage to separate the trachea. An inverted T-shaped incision was created, through which a Y-shaped tracheal tube was inserted and retained to ensure unobstructed breathing (the base respiration rate was increased by $10 \%$, and the tidal volume was $10 \mathrm{~mL} / \mathrm{kg}$ ). One side of the common carotid artery was separated, and the arterial catheter was inserted through it. The catheter was divided into two channels through a three-way valve: one channel was connected to the BL-420F bio-signal acquisition and analysis system to record blood pressure [including systolic blood pressure (SBP), diastolic blood pressure (DBP), and mean arterial pressure (MAP)] and heart rate (HR) (the arterial catheter and pressure sensor catheter filled with heparin before the intubation to prevent occlusion of blood pressure pathway after coagulation); the other channel was clamped to avoid arterial blood loss and for blood sampling. Bloodletting was not performed in the Sham group.

The rabbits in Shock group and fluid infusion groups experienced quicker bloodletting to reduce the MAP to 40$50 \mathrm{mmHg}$ within 10 minutes by loosening the carotid artery catheter clip, which showed significant blood pressure reduction and metabolic acidosis, demonstrating the occurrence of hemorrhagic shock. The status of shock was maintained throughout the research in the Shock group. In the operation with fluid infusion groups, the status of shock was maintained for 20 minutes; subsequently, the fluid was infused at a rate of $60 \mathrm{~mL} / \mathrm{kg}$ per hour via the auricular vein (punctured by using a scalp needle) for 1.5 hours. The fluids used in all groups had the same appearance. Both the experimenters and the statistical analyzers were blind to the grouping, which ensured randomization and double blinding. Unblinding was performed after the experiments were completed.

\section{Determination indicators and methods}

\section{Vital signs}

During the experiment, changes in HR, SBP, DBP, and MAP were continuously monitored at all observation point: before shock (baseline, T0); infusion for 0 minutes (T1, 20 minutes after shock), 30 minutes (T2), 60 minutes (T3), and 90 minutes (T4); and 30 minutes (T5) and 60 minutes (T6) after infusion.

\section{Blood gas analysis}

Fresh anticoagulated whole blood samples were collected for detection of $\mathrm{pO}_{2}, \mathrm{pCO}_{2}, \mathrm{pH}, \mathrm{HCO}_{3}^{-}$, base excess (BE), lactic acid (Lac) at five time points: T0, T1, T3, T5, T6.

\section{Blood biochemistry}

Serum was collected at the T0, T1, T5 and T6 for determining creatinine $(\mathrm{Cr})$, urea nitrogen (BUN), alanine aminotransferase (ALT), and aspartate aminotransferase (AST) levels.

\section{Analysis of intestinal flora}

Feces were collected upon the end of the experiments for $16 \mathrm{~S}$ ribosomal RNA (rRNA) gene sequencing. The analysis included sequencing [including DNA extraction, polymerase chain reaction (PCR) amplification, MiSeq library construction, and MiSeq sequencing] and bioinformatic analysis (including operational taxonomic unit clustering, alpha-diversity index, dilution curve analysis, and taxa heatmap).

\section{Histopathological examinations of lung tissues}

The lung tissue was fixed with $10 \%$ formalin, conventionally dehydrated, embedded in paraffin and cut into serial sections, which were subject to HE staining and observed and photographed under a microscope (magnification, $\times 200$ ). Lung injury was evaluated based on a modified scoring system consisting of five categories: 
alveolar structure, alveolar septum, neutrophil infiltration, alveolar hemorrhage, and alveolar edema. Each category was classified as none, mild, moderate, or severe, and scored as $0,1,2$, and 3 points, respectively. Five visual fields of each tissue section were selected randomly under a microscope at high magnification, and the average value was obtained as the lung tissue injury score.

\section{TUNEL staining of liver and kidney tissues for detecting cell apoptosis}

Liver and kidney tissues were taken for routine paraffinembedding and sectioning. After staining according to the manufacturer's instructions for the TUNEL cell apoptosis kit, the number of apoptotic cells was observed under a light microscope. The cells with brown particles in the nucleus were the apoptotic cells. Under a high-power microscope (magnification, $\times 200$ ), five fields of vision were randomly collected for counting the positive cells. The apoptosis index was calculated as the number of apoptotic cells divided by the total number of cells counted.

\section{Statistical analysis}

The measurement data are presented as mean \pm standard deviation. The statistical analyses were performed by using the GraphPad Prism software (GraphPad Software, Inc., San Diego, CA, USA). Vital signs, blood gas analysis and blood biochemistry were analyzed using repeated measures ANOVA analysis and the Bonferroni correction was used to do multiple comparisons. Pathological sections of lung tissue and apoptosis of renal and hepatic cells were tested by $t$ test. A $\mathrm{P}$ value $<0.05$ was regarded as a statistically significant difference.

\section{Results}

\section{Vital signs}

At T1, compared with the Sham group, the Shock group and the infusion groups had significantly decreased MAP, SBP, and DBP; in addition, the blood pressure gradually fell with time in the Shock group. Compared with the Shock group, the decreased blood pressure in the infusion groups was significantly improved, and there were no significant differences among the three infusion groups (Figure 1, Table 2).

\section{Findings of blood gas analysis}

Compared with the Sham group, the blood $\mathrm{pH}, \mathrm{BE}$, and $\mathrm{HCO}_{3}{ }^{-}$levels in the Shock group were significantly reduced $(\mathrm{P}<0.05, \mathrm{P}<0.01)$, while the lactic acid level was significantly increased $(\mathrm{P}<0.01)$, showing a significant acid-base imbalance; the acid-base imbalance was improved in all three of the fluid resuscitation groups. The reduction in both $\mathrm{BE}$ $\mathrm{HCO}_{3}{ }^{-}$caused by acidosis in the rabbits with hemorrhagic shock were significantly improved in the BRS group when compared with Saline group at T3 and T5 (BRS group $v s$. Saline Group: BE: at T3, $-4.83 \pm 3.60$ vs. $-12.50 \pm 3.27 \mathrm{mmol} / \mathrm{L}$, $\mathrm{P}<0.01$; at $\mathrm{T} 5,-3.67 \pm 4.37$ vs. $-11.00 \pm 2.76 \mathrm{mmol} / \mathrm{L}$, $\mathrm{P}<0.01 ; \mathrm{HCO}_{3}^{-}$: at T3, $22.15 \pm 2.63$ vs. $15.42 \pm 3.03 \mathrm{mmol} / \mathrm{L}$, $\mathrm{P}<0.01$; at $\mathrm{T} 5,23.15 \pm 2.9$ vs. $16.23 \pm 3.07 \mathrm{mmol} / \mathrm{L}, \mathrm{P}<0.01$ ). Compared with ARS group, the BRS group tended towards an improved acid-base imbalance, although the difference was not statistically significant. At T3, compared with the Saline group, the BRS group had significantly lower $\mathrm{pO}_{2}$ level $(\mathrm{P}<0.01)$, possibly due to the BRS more improving the acidosis of the shocked rabbits and the oxygen dissociation curve being shifted to the left (Figure 2, Table 2).

\section{Results of blood biochemistry}

Compared with the Sham group, the renal function indicators including creatinine and urea nitrogen were significantly increased in the Shock and infusion groups (all $\mathrm{P}<0.05$ ), and there were no significant differences in renal function indicators between the Shock group and the infusion groups.

ALT showed no significant difference among the five groups. The AST showed an upward trend with prolonged shock time in the Shock group and Saline group. In the ARS and BRS groups, AST showed a downward trend after fluid resuscitation. Analysis of $\triangle \mathrm{AST}$ (T1-T5) and $\triangle$ AST (T1-T6) showed $\triangle$ AST (T1-T6) to be significantly different between the Saline group and BRS group $(28.49 \pm 25.78$ vs. $-7.81 \pm 8.96 \mathrm{U} / \mathrm{L}, \mathrm{P}<0.01)$. Resuscitation with normal saline increased AST in the hemorrhagic shock models, while resuscitation with BRS improved the increase in AST caused by hemorrhagic shock (Figure 3), although the mechanism behind this was unclear.

\section{Results of intestinal flora analysis}

Compared with the Sham group, the Shock group had ten 

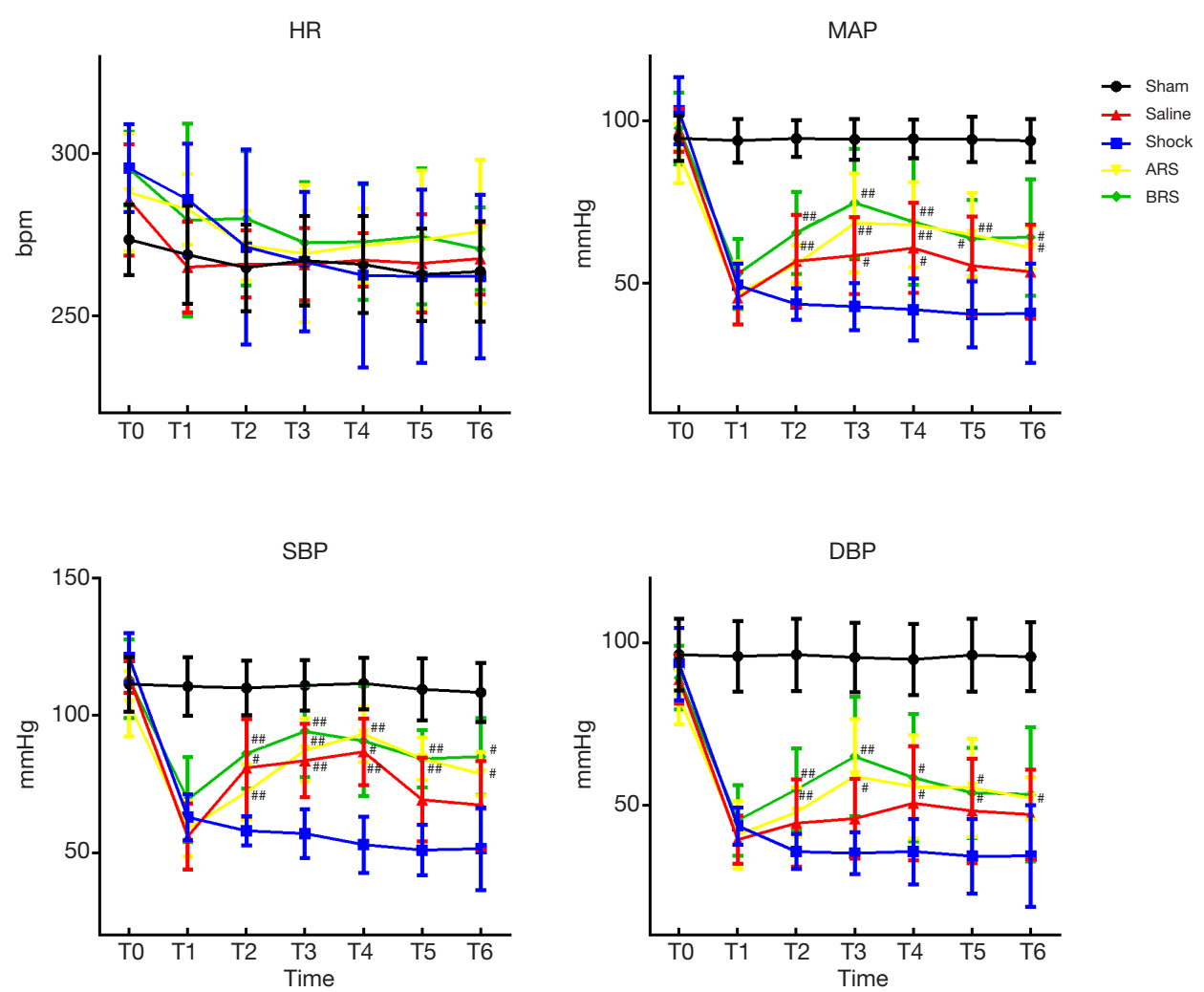

Figure 1 Vital signs in five groups. The decline of MAP, DBP, and SBP caused by hemorrhagic shock was significantly improved in the infusion groups. ", $\mathrm{P}<0.05$ and ${ }^{\# \#}, \mathrm{P}<0.01$ compared with the Shock group. HR, heart rate; MAP, mean arterial pressure; SBP, systolic blood pressure; DBP, diastolic blood pressure; ARS, acetated Ringer's solution; BRS, bicarbonated Ringer's solution; T0, before shock (baseline); $\mathrm{T} 1$, infusion for 0 minutes (20 minutes after shock); T2, infusion for 30 minutes; T3, infusion for 60 minutes; T4, infusion for 90 minutes; T5, 30 minutes after infusion; T6, 60 minutes after infusion.

differential microbiotas, indicating that the Shock group had severe intestinal flora disorder; meanwhile, the ARS group and the Sham group had five differential microbiotas, indicating that intestinal flora disorder also occurred in the ARS group but was improved to a certain extent. There were three differential microbiotas between the BRS group and the Sham group, suggesting that BRS was superior to ARS in improving intestinal flora disorder in shock rabbits (Figure 4).

\section{Pathological sections of lung tissue}

Compared with the Sham group, the Shock group had slight changes in alveolar structure and alveolar septum, increased neutrophil infiltration in the lung tissue, and a small amount of hemorrhage and mild edema in the alveolar cavity. Compared with the Shock group, the three infusion groups had improved pathological changes of the lung tissue (Figure 5). The results of pathological scoring are shown in Table 3 and Figure 6.

\section{Apoptosis of renal and hepatic cells}

The apoptosis of renal and hepatic cells is shown in Figure 5. Compared with the Sham group, the amount of apoptotic cells in the liver and kidney tissues significantly increased in the Shock group $(\mathrm{P}<0.01)$. Compared with the Shock group, fluid resuscitation attenuated the apoptosis of renal and hepatic cells in the Saline group, ARS group, and BRS group to varying degrees (Table 4), with the liver apoptosis index in the ARS group and the BRS group significantly differing from that in the Shock group (both $\mathrm{P}<0.05$ ).

\section{Discussion}

At present, the commonly used isotonic crystalloids include 
Table 2 Vital signs, blood gas analysis results, and liver function in the five groups (mean \pm SD, n=6)

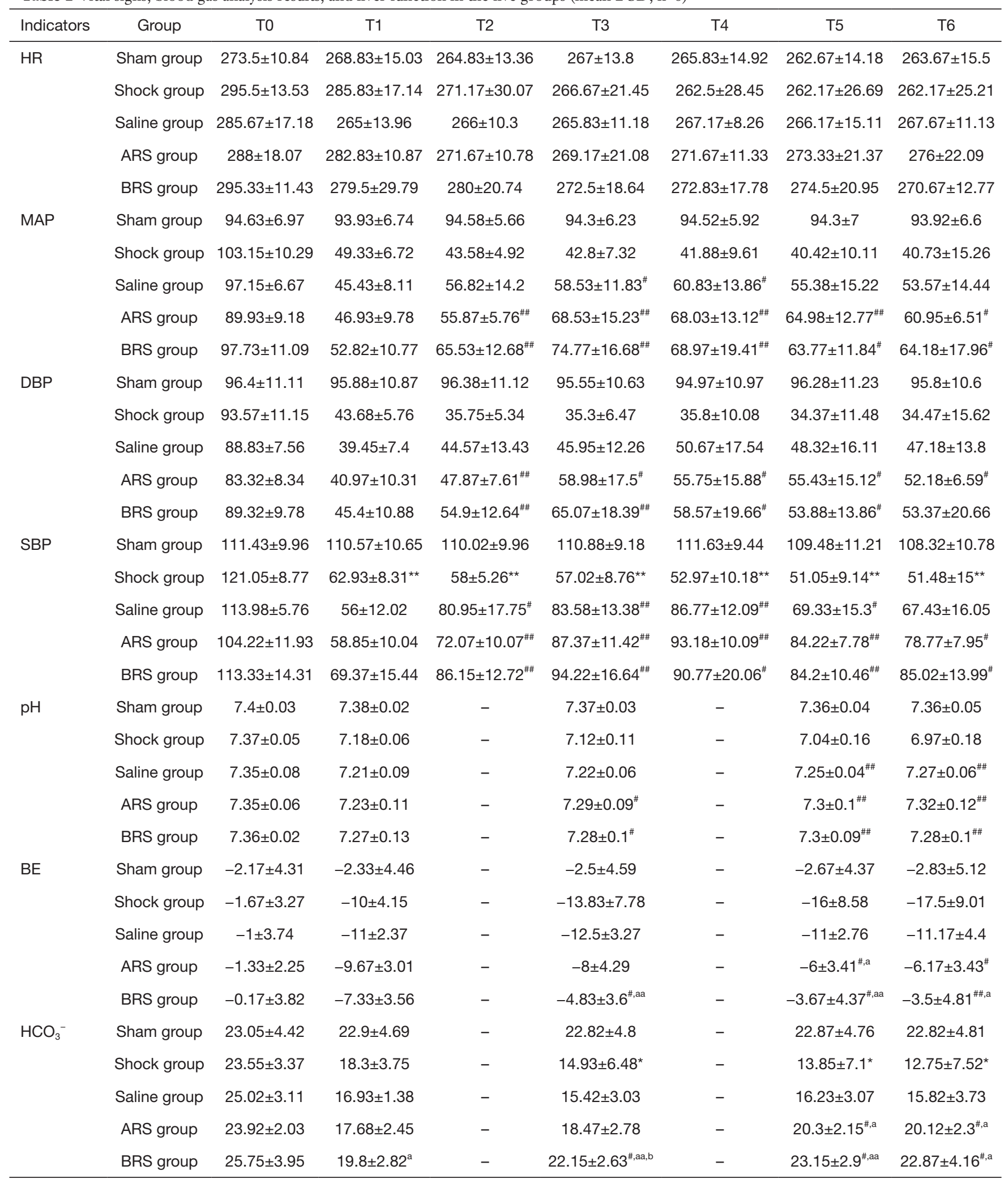

Table 2 (continued) 
Table 2 (continued)

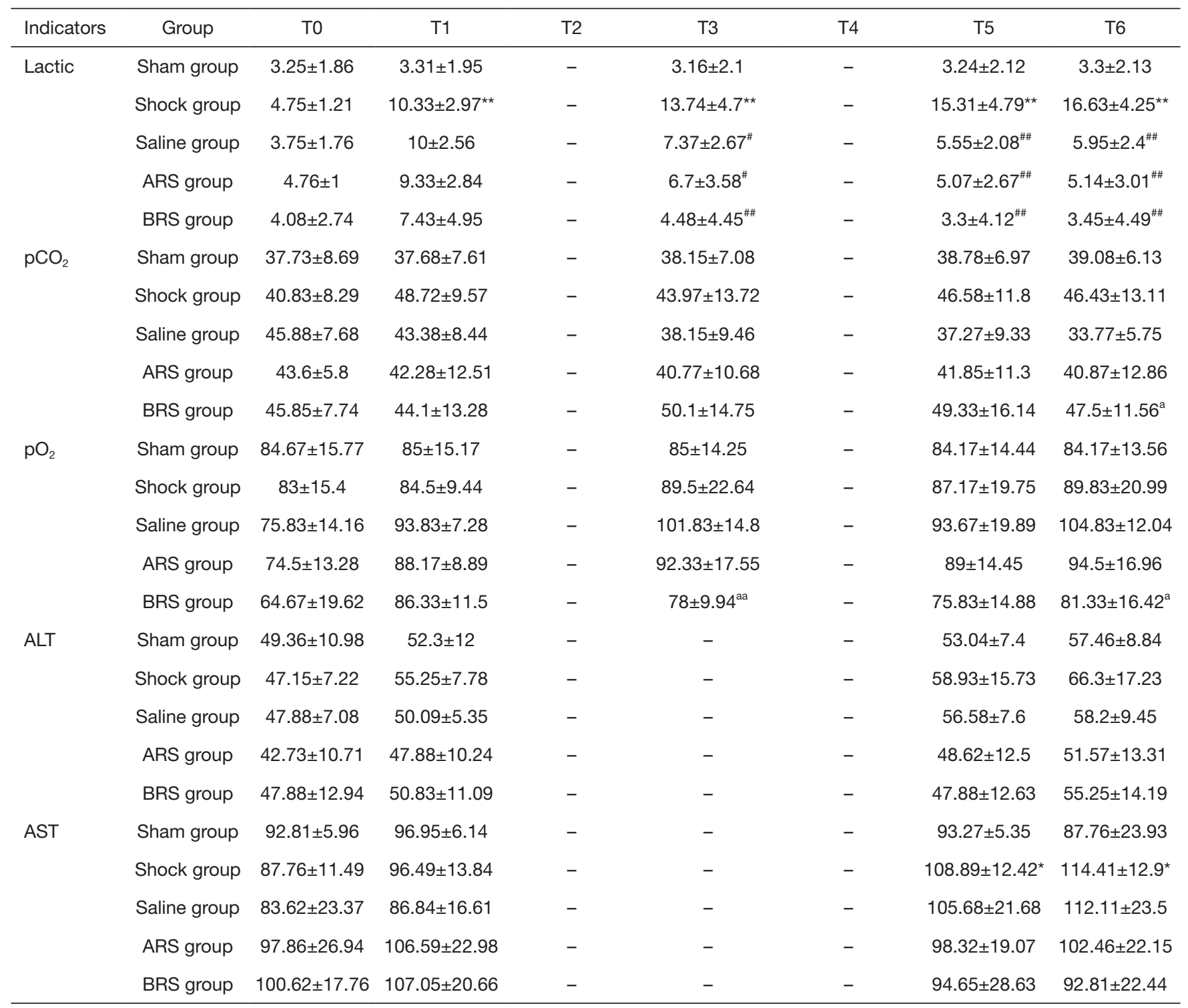

*, $\mathrm{P}<0.05$ and ${ }^{*}, \mathrm{P}<0.01$ compared with the Sham group; ${ }^{\#}, \mathrm{P}<0.05$ and ${ }^{\# \#}, \mathrm{P}<0.01$ compared with the Shock group; ${ }^{\text {a }}, \mathrm{P}<0.05$ and ${ }^{\text {aa }}$, $\mathrm{P}<0.01$ compared with the Saline group; ${ }^{b}, \mathrm{P}<0.05$ compared with the ARS group. HR, heart rate; MAP, mean arterial pressure; SBP, systolic blood pressure; DBP, diastolic blood pressure; $\mathrm{BE}$, base excess; $\mathrm{HCO}_{3}{ }^{-}$, bicarbonate; $\mathrm{PCO}_{2}$, partial pressure of carbon dioxide; $\mathrm{pO}_{2}$, partial pressure of oxygen; ALT, alanine aminotransferase; AST, aspartate aminotransferase; ARS, acetated Ringer's solution; BRS, bicarbonated Ringer's solution. T0, before shock (baseline); T1, infusion for 0 minutes (20 minutes after shock); T2, infusion for 30 minutes; T3, infusion for 60 minutes; T4, infusion for 90 minutes; T5, 30 minutes after infusion; T6, 60 minutes after infusion.

normal saline, lactated Ringer's solution (LRS), and acetated Ringer's solution (ARS). The chloride ion concentration in normal saline is higher than the normal physiological concentration, with a strong ion difference (SID) of 0 , and thus a massive infusion of normal saline can lead to hyper chloric acidosis (11) and even kidney injury (5). LRS and
ARS are added with alkalizing components (sodium lactate and sodium acetate, respectively), which can be converted into bicarbonate in the body to alkalinize the blood and thus correct metabolic acidosis (12). Up to $80-90 \%$ of the lactate in LRS is metabolized in the liver. A mass infusion of LRS in patients with liver dysfunction may cause lactic acid 

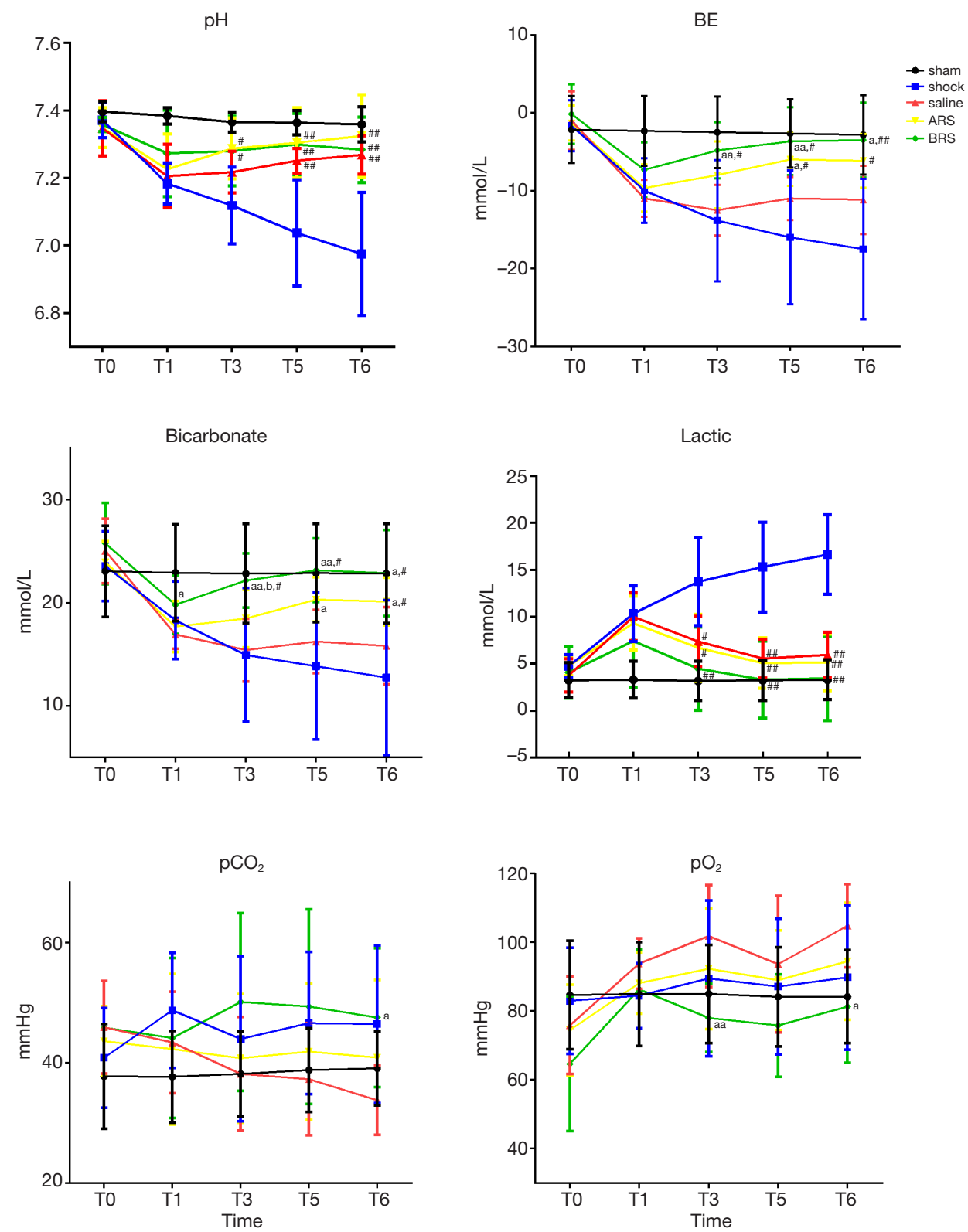

Figure 2 Arterial blood gases in five groups. ", $\mathrm{P}<0.05$ and ${ }^{\# \#}, \mathrm{P}<0.01$ compared with the Shock group. ${ }^{\text {a }}, \mathrm{P}<0.05$ and ${ }^{\text {aa }}, \mathrm{P}<0.01$ compared with the Saline group; ${ }^{b}, \mathrm{P}<0.05$ compared with the ARS group. The metabolic acidosis caused by hemorrhagic shock was significantly improved in the infusion groups. The improvements in $\mathrm{BE}$ and $\mathrm{HCO}_{3}{ }^{-}$were more significant in the $\mathrm{BRS}$ group than in the Saline group. $\mathrm{BE}$, base excess; $\mathrm{pCO}_{2}$, partial pressure of carbon dioxide; $\mathrm{pO}_{2}$, partial pressure of oxygen; ARS, acetated Ringer's solution; BRS, bicarbonated Ringer's solution. T0, before shock (baseline); T1, infusion for 0 minutes (20 minutes after shock); T3, infusion for 60 minutes; T5, 30 minutes after infusion; T6, 60 minutes after infusion.

accumulation (8). Half (50\%) of the acetate in ARS is also metabolized by the liver (6), and part of it is metabolized by other tissues such as the kidneys and muscles. Acetate has a vasodilator effect, and a massive infusion of ARS may cause vasodilation, leading to hypotension (13). BRS is the latest generation of balanced crystalloid solutions that contains physiological concentrations of bicarbonate ions, which can quickly combine with excess hydrogen ions in the body to 

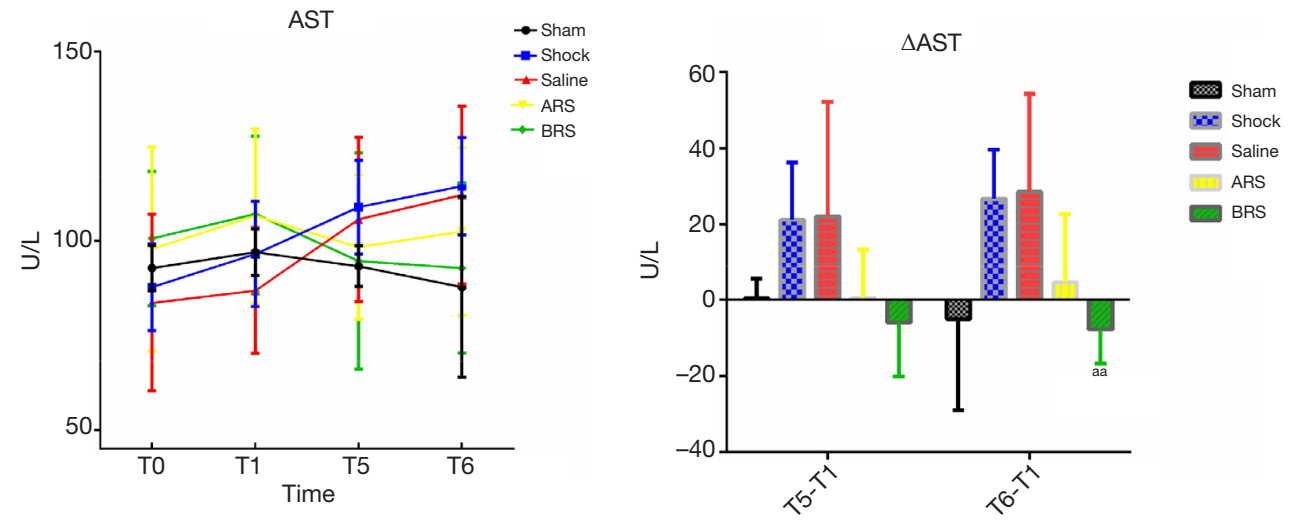

Figure 3 AST and $\triangle$ AST in five groups. ${ }^{\text {aa }}, \mathrm{P}<0.01$ compared with the Saline group. The increased AST caused by hemorrhagic shock was significantly improved in the BRS group. AST, aspartate aminotransferase; ARS, acetated Ringer's solution; BRS, bicarbonated Ringer's solution. T0, before shock (baseline); T1, infusion for 0 minutes (20 minutes after shock); T5, 30 minutes after infusion; T6, 60 minutes after infusion.

A
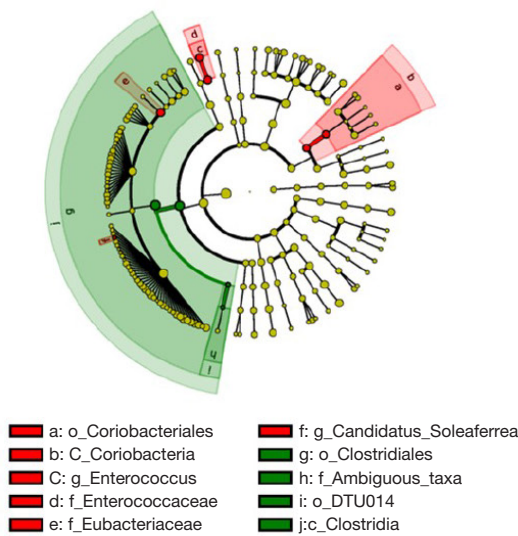

B
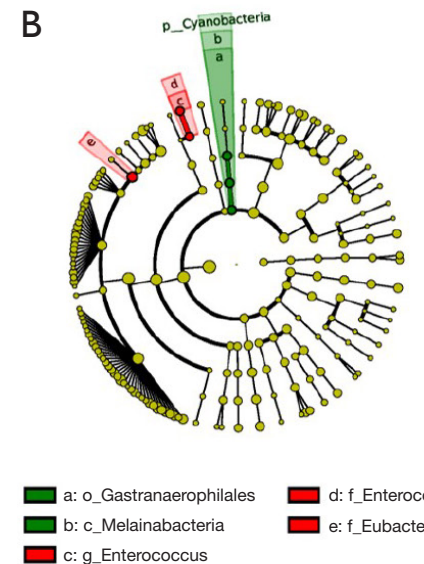

C

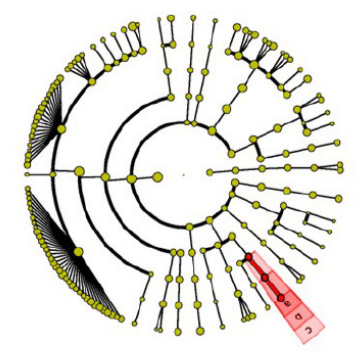

$\square$ a: g_Escherichia_Shigella $\square$ c: o_Enterobacteriales

Figure 4 Results of intestinal flora sequencing. (A) Compared with the Sham group, the Shock group had 10 differential microbiotas, indicating that the Shock group had severe intestinal flora disorder; (B) compared with the Sham group, the ARS group had 5 differential microbiotas, indicating that intestinal flora disorder also occurred in the ARS group but was improved to a certain extent; (C) there were 3 differential microbiotas between the BRS group and the Sham group, suggesting that BRS was superior to ARS in improving the intestinal flora disorder in shock rabbits. ARS, acetated Ringer's solution; BRS, bicarbonated Ringer's solution.

correct metabolic acidosis; in addition, the bicarbonate is not metabolized via the liver and thus will not overburden the liver. Research has shown that, compared with other crystalloid solutions, BRS can markedly correct the acidbase imbalance $(9,10)$. Crystalloid infusions to 3 liters in the first 6 hours after arrival at the hospital was recommended as part of a bundle of care for patients with acute bleeding from trauma (14); however, it did not specifically recommend which fluid should be applied. Few reports on the effects of resuscitation with different crystalloid solutions on hemorrhagic shock (especially on the tissues and organs) are available. In our current study, we compared the effects of normal saline, ARS, and BRS for resuscitation on the internal environments and tissues/organs of the hemorrhagic shock models. The age, body weight, and baseline status were matched among the five groups in order to minimize selection bias. It was found that, compared with the normal saline, BRS could significantly improve the 

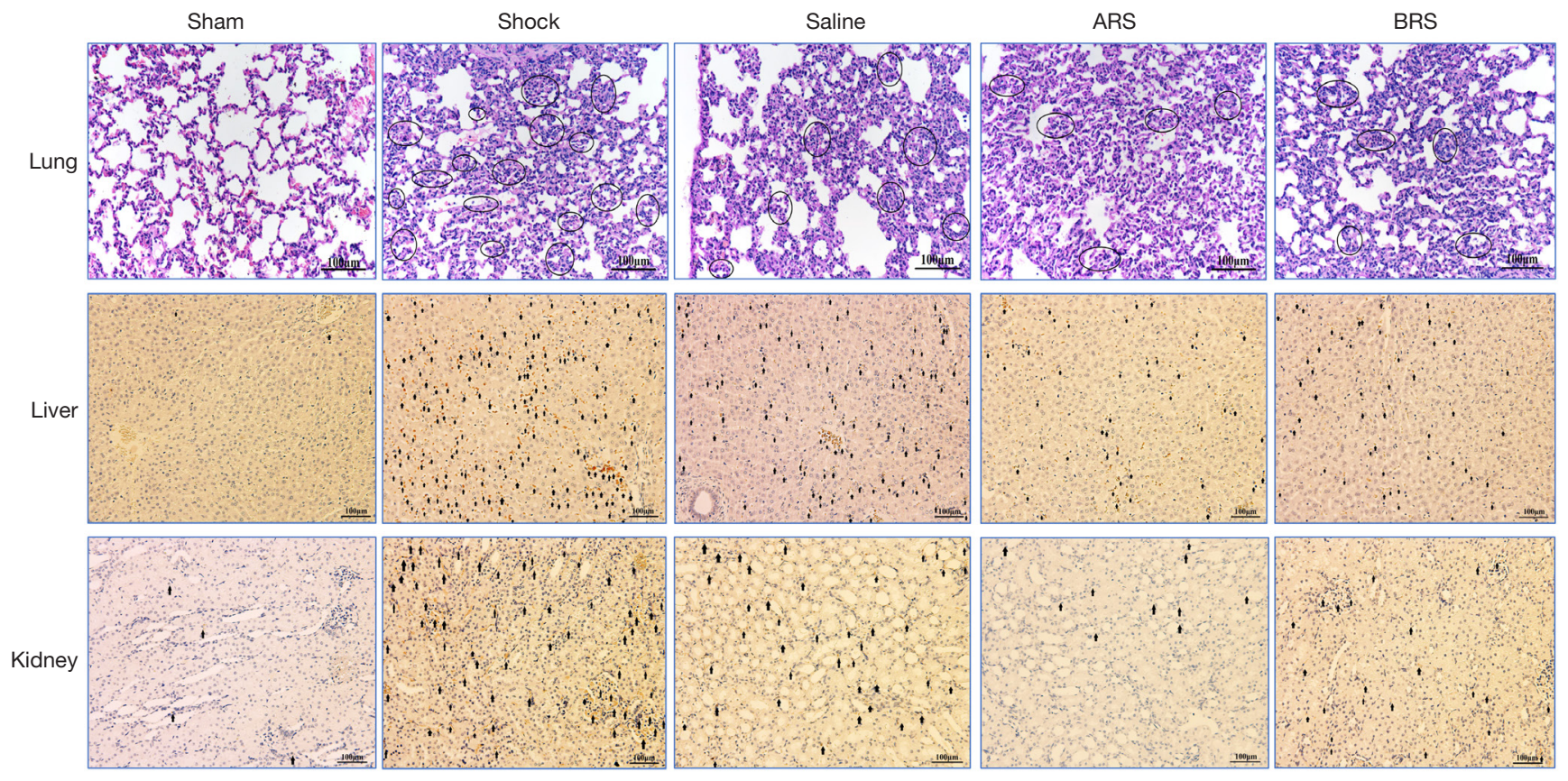

Figure 5 Pathological sections of the lung tissue by hematoxylin-eosin staining, apoptosis of renal and hepatic cells by TdT-mediated dUTP Nick-End Labeling in five groups (magnification $\times 200$ ). Compared with the Shock group, the pathological changes of the lung tissue in the three infusion groups had improved. Apoptosis of renal and hepatic cells in rabbits with hemorrhagic shock were improved in the Saline group, ARS group, and BRS group. The improvements in the ARS group and BRS group were statistically significant when compared with the Shock group (both $\mathrm{P}<0.05$ ). The location of neutrophil infiltration has been marked by black circle. And the apoptosis of renal and hepatic cells has been marked by black arrow. ARS, acetated Ringer's solution; BRS, bicarbonated Ringer's solution.

Table 3 Effect of fluid resuscitation on the histopathological score of lung injury in rabbits with hemorrhagic shock (mean \pm SD, $n=6)$

\begin{tabular}{|c|c|c|c|c|c|c|}
\hline Group & Alveolar structure & Alveolar septum & Neutrophil infiltration & Alveolar hemorrhage & Alveolar edema & Total score \\
\hline Shock group & $1.00 \pm 0.00$ & $1.00 \pm 0.00$ & $1.83 \pm 0.41$ & $1.17 \pm 0.41$ & $0.33 \pm 0.52$ & $5.33 \pm 1.03$ \\
\hline Saline group & $1.00 \pm 0.00$ & $1.00 \pm 0.00$ & $2.00 \pm 0.89$ & $0.67 \pm 0.52$ & $0.00 \pm 0.00$ & $4.67 \pm 1.37$ \\
\hline ARS group & $1.00 \pm 0.00$ & $1.00 \pm 0.00$ & $1.50 \pm 0.55$ & $1.00 \pm 0.00$ & $0.00 \pm 0.00$ & $4.50 \pm 0.55$ \\
\hline
\end{tabular}

ARS, acetated Ringer's solution; BRS, bicarbonated Ringer's solution.

metabolic acidosis caused by hemorrhagic shock, bringing the $\mathrm{BE}$ and $\mathrm{HCO}_{3}^{-}$levels of the rabbits with hemorrhagic shock closer to those of the Sham group (normal levels). The BRS group showed a superior tendency in improving acidosis than did the ARS group, although the difference was not statistically significant. Hemorrhagic shock causes tissue and organ damage, leading to the release of AST into the bloodstream. BRS significantly attenuated the increase in AST caused by hemorrhagic shock, indicating that BRS may have a certain protective effect on tissues and organs. AST is mainly distributed in the myocardium, followed by the liver, skeletal muscle, and kidneys. However, which organs can be specifically protected by BRS needs to be further explored. Both ARS and BRS improved the liver cell apoptosis caused by shock and have certain protective effects on the liver; notably, this effect was not obvious in the Saline group.

Generally, a decrease in MAP will lead to a compensatory 


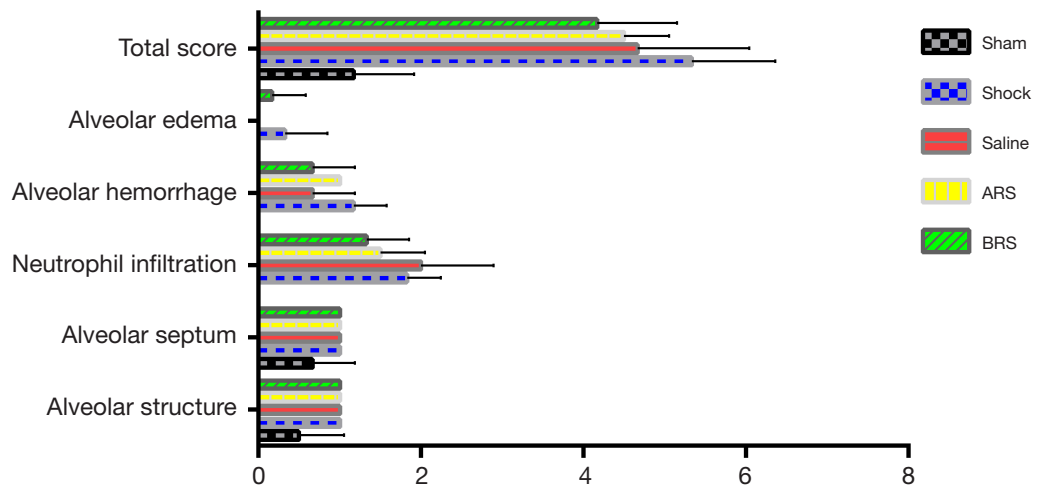

Figure 6 Histopathological score of lung injury in five groups. Compared with the Shock group, the total score of the lung tissue in the three infusion groups had improved, but no significant difference. ARS, acetated Ringer's solution; BRS, bicarbonated Ringer's solution.

Table 4 Effect of fluid resuscitation on the apoptosis of renal and hepatic cells in rabbits with hemorrhagic shock (mean $\pm S D, n=6$ )

\begin{tabular}{lcc}
\hline Group & $\begin{array}{c}\text { Liver apoptosis index } \\
(\%)\end{array}$ & $\begin{array}{c}\text { Kidney apoptosis index } \\
(\%)\end{array}$ \\
\hline Sham group & $9.9 \pm 1.1$ & $8.3 \pm 1.2$ \\
Shock group & $28.1 \pm 6.1$ & $21.2 \pm 6.8$ \\
Saline group & $20.7 \pm 5.9$ & $20.0 \pm 3.4$ \\
ARS group & $19.8 \pm 5.4^{\#}$ & $18.0 \pm 5.9$ \\
BRS group & $19.1 \pm 3.3^{\#}$ & $17.0 \pm 3.5$ \\
\hline
\end{tabular}

\#, $\mathrm{P}<0.05$ compared with the Shock group. ARS, acetated Ringer's solution; BRS, bicarbonated Ringer's solution.

increase in HR, however, we did not observe an increase trend in HR at T1. It might be because the HR has passed the initial compensatory increase stage after 20 minutes of blood loss. At this time, as seen by the results of a significant increase in lactate, tissue has been significantly hypoxic, and the systolic and diastolic function of the myocardium has also been inhibited.

The strength of our current study was its randomized double-blind design, which minimized possible biases. Using different fluids in the hemorrhagic shock models, we explored the effects of early resuscitation on the internal environment and organ functions, the findings from which may help inform the clinical selection of crystalloid solutions.

However, this study also had certain limitations: the rabbits in this study had short survival time, and the final observation session was performed only 2.5 hours after shock; as a result, some indicators such as AST, ALT, BUN,
$\mathrm{Cr}$, and changes in intestinal flora were not adequately expressed, so that these indicators did not show a significant change trend. Therefore, animals with longer survival will be used in our future investigations.

In summary, when used for resuscitation of rabbits with traumatic hemorrhagic shock, BRS can markedly improve metabolic acidosis caused by hemorrhagic shock, maintain the acid-base balance, and, to varying degrees, improve organ and tissue damage caused by hemorrhagic shock. Thus, BRS may be an effective balanced crystalloid solution for the resuscitation of hemorrhagic shock. However, our current study was performed in rabbit models of hemorrhagic shock, and whether or not these findings can be applied in clinical settings remains to be further studied.

\section{Acknowledgments}

Funding: Funding was provided by Jiangsu Hengrui Pharmaceutical Co., Ltd.

\section{Footnote}

Reporting Checklist: The authors have completed the ARRIVE reporting checklist. Available at http://dx.doi. org/10.21037/atm-21-97

Data Sharing Statement: Available at http://dx.doi. org/10.21037/atm-21-97

Conflicts of Interest: All authors have completed the ICMJE uniform disclosure form (available at http://dx.doi. org/10.21037/atm-21-97). All authors report that grants 
were from Jiangsu Hengrui Pharmaceutical Co., Ltd., outside the submitted work.

Ethical Statement: The authors are accountable for all aspects of the work in ensuring that questions related to the accuracy or integrity of any part of the work are appropriately investigated and resolved. This study was approved by the Ethical Committee of China Pharmaceutical University, Nanjing University, and Laboratory Animal Management Committee of Jiangsu Province (approval No. CPU-20190723). All of the animal experiments were conducted in compliance with the standard ethical guidelines under the control of the ethical committees mentioned above.

Open Access Statement: This is an Open Access article distributed in accordance with the Creative Commons Attribution-NonCommercial-NoDerivs 4.0 International License (CC BY-NC-ND 4.0), which permits the noncommercial replication and distribution of the article with the strict proviso that no changes or edits are made and the original work is properly cited (including links to both the formal publication through the relevant DOI and the license). See: https://creativecommons.org/licenses/by-nc-nd/4.0/.

\section{References}

1. Kauvar DS, Wade CE. The epidemiology and modern management of traumatic hemorrhage: US and international perspectives. Crit Care 2005;9 Suppl 5:S1-9.

2. Spinella PC, Holcomb JB. Resuscitation and transfusion principles for traumatic hemorrhagic shock. Blood Rev 2009;23:231-40.

3. Cannon JW. Hemorrhagic shock. N Engl J Med 2018;378:370-9.

4. Neal MD, Hoffman MK, Cuschieri J, et al. Crystalloid

Cite this article as: Wang L, Lou J, Cao J, Wang T, Liu J, Mi W. Bicarbonate Ringer's solution for early resuscitation in hemorrhagic shock rabbits. Ann Transl Med 2021;9(6):462. doi: 10.21037/atm-21-97 to packed red blood cell transfusion ratio in the massively transfused patient: when a little goes a long way. J Trauma Acute Care Surg 2012;72:892-8.

5. Semler MW, Self WH, Rice TW. Balanced crystalloids versus saline in critically ill adults. N Engl J Med 2018;378:1951.

6. Smyth DH. The rate and site of acetate metabolism in the body. J Physiol 1947;105:299-315.

7. Adeva-Andany M, López-Ojén M, Funcasta-Calderón $\mathrm{R}$, et al. Comprehensive review on lactate metabolism in human health. Mitochondrion 2014;17:76-100.

8. Marko P, Gabrielli A, Caruso LJ. Too much lactate or too little liver? J Clin Anesth 2004;16:389-95.

9. Satoh K, Ohtawa M, Katoh M, et al. Pharmacological study of BRS, a new bicarbonated Ringer's solution, in haemorrhagic shock dogs. Eur J Anaesthesiol 2005;22:703-11.

10. Nakayama M, Yamauchi M, Kanaya N, et al. Utility of bicarbonated Ringer's solution as an intraoperative fluid during long-term laparotomy. Masui 2007;56:1334-8.

11. Morgan TJ, Venkatesh B, Hall J. Crystalloid strong ion difference determines metabolic acid-base change during acute normovolaemic haemodilution. Intensive Care Med 2004;30:1432-7.

12. Pfortmueller CA, Fleischmann E. Acetate-buffered crystalloid fluids: Current knowledge, a systematic review. J Crit Care 2016;35:96-104.

13. Hartsfield SM, Thurmon JC, Corbin JE, et al. Effects of sodium acetate, bicarbonate and lactate on acid-base status in anaesthetized dogs. J Vet Pharmacol Ther 1981;4:51-61.

14. Shafi S, Collinsworth AW, Richter KM, et al. Bundles of care for resuscitation from hemorrhagic shock and severe brain injury in trauma patients-Translating knowledge into practice. J Trauma Acute Care Surg 2016;81:780-94.

(English Language Editor: J. Gray) 